\title{
Body System Code
}

National Cancer Institute

\section{Source}

National Cancer Institute. Body System Code. NCI Thesaurus. Code C93522.

A coded value specifying the anatomical grouping that consists of organs and organ subclasses that are together responsible for certain body functions. 